\title{
Entre el saqueo y la restitución: el caso Susana Soca
}

\section{Valentina Litvan}

\section{OpenEdition}

\section{Journals}

Edición electrónica

URL: https://journals.openedition.org/cher/3079

DOI: 10.4000/cher.3079

ISSN: 2803-5992

\section{Editor}

Presses universitaires de Strasbourg

\section{Edición impresa}

Fecha de publicación: 27 junio 2017

ISBN: 978-2-86820-961-0

ISSN: 1968-035X

\section{Referencia electrónica}

Valentina Litvan, «Entre el saqueo y la restitución: el caso Susana Soca», reCHERches [En línea], 18 | 2017, Publicado el 01 diciembre 2021, consultado el 17 diciembre 2021. URL: http:// journals.openedition.org/cher/3079 ; DOI: https://doi.org/10.4000/cher.3079

\section{๑๑॰}

$\mathrm{Ce}(\mathrm{tte})$ œuvre est mise à disposition selon les termes de la Licence Creative Commons Attribution -

Pas d'Utilisation Commerciale - Partage dans les Mêmes Conditions 4.0 International. 


\title{
Entre el saqueo y la restitución: el caso Susana Soca
}

\author{
Valentina LITVAN*
}

$\mathrm{E}$

ntre el saqueo y la restitución, la presencia de Susana Soca es ambivalente dentro de la tradición cultural uruguaya: Susana Soca es y no es autora, si aparece en el canon literario es siempre desde afuera, como extranjera o por su aporte extranjerizante; en tanto editora es ante todo una lectora, que presenta los textos de los autores a los que publica, y su mecenazgo fluctúa entre la iniciativa privada posibilitada por su enorme fortuna personal y la legitimación pública al contar con el apoyo institucional. Susana Soca es asimismo musa, o mejor cabría decir fuente de inspiración para distintos escritores, objeto de dedicatorias literarias y de obras plásticas; pero por encima de todo ello, Susana Soca se va perfilando como una construcción de terceros, convirtiéndose al fin ella misma en ficción.

Mi hipótesis es que la intertextualidad en el caso particular de Susana Soca está vinculada ya no al sentido originario que pudieran dar Mijaíl Bajtín o Julia Kristeva de diálogos, préstamos, absorciones o transformaciones textuales, sino más bien a las trasposiciones que operan en el propio fenómeno literario haciendo trastabillar cualquier tipo de categoría única y estable. De la intertextualidad, se podría decir, a los desplazamientos ocurridos en el interior mismo de la cadena de producción literaria, donde categorías como autor-obralector no configuran una serie unidireccional ni exclusiva, pues a ellas se les añaden otras (editor, lector, mecenas, musa, ficción, hemos dicho), que lejos de quedar delimitadas se nutren unas a otras, se superponen, se confunden: Susana Soca encarna esa compleja trama del proceso creativo. El lugar ambivalente y fluctuante que ocupa en la tradición invita a cuestionarnos entonces sobre el propio fenómeno literario, como trataré de demostrar.

* Profesor titular y miembro del Centro de Investigación Ecritures EA3943 en la Université de Lorraine - France. 
Para hacer comprensible esta hipótesis, recorreré paralelamente la trayectoria biográfica y literaria de una figura que, aunque clave en el contexto cultural del Uruguay de los años 50, sigue siendo para muchos una desconocida ${ }^{1}$.

Susana Soca nació en Montevideo en 1907, heredando una gran fortuna y apellidos de la alta sociedad de su país. Por parte de su madre está vinculada a los Blanco y Acevedo, apellidos de fuerte impronta en la historia política y cultural uruguaya: así, por ejemplo, la novela histórica nacional fue fundada por un tío abuelo suyo, Eduardo Acevedo Díaz²; su abuelo, Juan Carlos Blanco, fue el opositor de Batlle y Ordóñez para la candidatura como presidente del Partido Colorado que asume el poder en 1903; y uno de sus tíos por vía materna, médico, recibió la Legión de Honor de manos del general De Gaulle.

En cuanto a su padre, Francisco Soca, fue médico y miembro del Ateneo de Montevideo, que era el centro de ebullición cultural de principios del siglo xx, donde se expresaban las ideas filosóficas del momento, y lugar de encuentro entre hombres de letras, científicos y políticos responsables del proyecto para un Uruguay que definían por su "carácter democrático» y el «cosmopolitismo de las ciencias» ${ }^{3}$. Como muchos otros médicos y abogados a caballos de siglo, el doctor Soca siguió sus estudios en París, convirtiéndose en el primer sudamericano Miembro de la Academia de Medicina de París ${ }^{4}$. Es en la capital europea donde decide bautizar a Susana Soca en 1908, nada menos que en la catedral de Notre Dame de París, gesto que se instaura como signo de un destino, pues el vínculo con París es determinante tanto en la biografía como en la existencia dentro del campo literario para Susana Soca.

Es precisamente en la capital francesa donde, en 1938, varios años tras la muerte de su padre, Soca se instala con su madre. Lo que en principio era un paseo, se convirtió en una experiencia fundamental, ya que al estallar la guerra y con la Ocupación, la estadía se prolongó casi una década. Durante esos años, en tanto uruguaya afortunada y aventajada por el hecho de pertenecer a un país

1 Prueba de ello es el título del primer y único libro durante largos años dedicado a la autora: Juan Álvarez Márquez, Susana Soca, esa desconocida, Linardi y Risso, Montevideo, 2001. Solo en 2012, Claudia Amengual publica Rara avis. Vida y obra de Susana Soca, Prisa Ediciones, Montevideo. Ambos libros se tratan de biografías con por momentos un tono ficcional, donde la imagen que ofrecen de Susana Soca es la de una mujer extraña o por lo menos, inaccesible y misteriosa. Sobre este tema, ver el capítulo 3 de mi tesis de doctorado, Susana Soca et le champ culturel uruguayen: projet, pratique et image d'écrivain, Universidad Paris 8, 2008.

2 El crítico uruguayo Alberto Zum Felde (1930: 278) escribe sobre Eduardo Acevedo Díaz: «Faltando en nuestra literatura el poema representativo del ciclo guerrero y gauchesco de nuestra historia, la novela histórica de Acevedo Díaz llena en cierto modo esa función, ya que, a la realidad histórica misma de sus elementos, aúna ese aliento epopéyico y legendario y el valor poemático de muchas de sus escenas.»

3 Eduardo Acevedo Díaz, «Propósitos intelectuales del Club Universitario», en http:// www.ateneodemontevideo.com/fundadores/eduardoacevedo.htm. Ver también al respecto Alberto Zum Felde (1930: 225-248).

4 Ver José María Estapé (1926). 
que se había mantenido neutral en el conflicto, Susana Soca pudo llevar a cabo su tarea de mecenas. Como lo fuera antes Jules Supervielle, poeta y amigo de Susana Soca, fue asignada, a partir de 1945, agregada a la Legación de Uruguay en París "para desempeñar funciones tendientes a lograr un mayor acercamiento espiritual y artístico entre Francia y Uruguay» ${ }^{5}$.

En tanto mecenas, tanto en Francia como sobre todo en Uruguay, Soca financia obras de arte y arquitectura, como por ejemplo, la construcción de una capilla del arquitecto vanguardista catalán Antoni Maria Bonet Castellana para el pueblo Soca, así llamado en homenaje a su padre, oriundo del lugar. Además, también viaja en búsqueda de autores y manuscritos valiosos. Así por ejemplo, Drieu La Rochelle (1992: 235) le entrega sus cuadernos para que los saque de la Francia en guerra: «Pourvu que Susana Soca veuille bien partir et sauver ces cahiers. L'écrivain persévère dans son être jusqu'à la fin ${ }^{6} . » O$ Boris Pasternak piensa en ella cuando se ve perseguido por el stalinismo ${ }^{7}$. Soca representa para estos escritores algo más que la supervivencia personal, se trata de la supervivencia de su obra y, con ella, de sus identidades de escritores.

Pero sobre todo, en el ambiente de bullicio intelectual que caracterizó la posguerra ${ }^{8}$, justo antes de volver al Uruguay, en 1947, funda en París los Cahiers de la Licorne, una revista que además de significar un espacio de encuentro para autores europeos silenciados o dispersados durante la guerra, permitió difundir a algunos escritores hispanoamericanos por primera vez en Europa (entre ellos, por ejemplo, publica las primeras traducciones de Ficciones de Jorge Luis Borges, un fragmento de Alturas de Machu Picchu de Pablo Neruda también publicado en Europa por primera vez en su revista o a Felisberto Hernández, a quien además de dar a conocer en Francia ya en esa época, financia su estadía europea $^{9}$ ). Susana Soca, de este modo, no solo publica a los autores, sino que también interviene en otros momentos del proceso de su producción literaria y artística, y bien hay que decir que los escritores supieron aprovecharse de su generosidad ${ }^{10} \ldots$

5 En Fondos de la Embajada de Uruguay en Francia.

6 Nota del 5 de junio de 1940.

7 Susana Soca (1966: 127-136) escribe un texto sobre su viaje a Moscú en busca de los manuscritos de Pasternak: «Encuentro y desencuentro». Ver también Guillermo de Torre (1959).

8 Gilles Vannier (1988: 69) habla de «bouillonnement intellectuel».

9 Así, además de publicar a Felisberto Hernández en La Licorne, lo ayuda económicamente durante su estadía en París y lo introduce en el Instituto Millington Drake de Londres, donde obtiene una beca de estadía.

10 La referencia a la remuneración económica de las colaboraciones no es superflua, pues se trataba de algo excepcional entre las publicaciones uruguayas. Muchos autores de la época se refieren a ello. Ricardo Paseyro explica que «Dados el prestigio y los medios de Susana, la clientela pululaba...» (Discurso en ocasión de la presentación del libro de Álvarez 2001 en la Universidad de Montevideo). Otro ejemplo nos lo ofrece Ida Vitale: «El peor enemigo de Susana y de su tarea fue aquella misma posibilidad económica. No se pensó que otros también la tenían, pero sin generosa 
En una segunda etapa, ya de regreso en su país de origen, Susana Soca funda en Montevideo nuevamente la revista que, a partir de 1953, ofrece al contrario la posibilidad de publicar en América Latina a autores franceses y de otros países europeos e invertir así la perspectiva en la relación de intercambio literario entre ambos continentes. La Licorne permite a Susana Soca convertirse en el nexo de una constelación de escritores entre los cuales, Roger Caillois, Paul Eluard, Jules Supervielle, Maurice Blanchot, Emile Cioran, Felisberto Hernández, Jorge Luis Borges, María Zambrano, José Bergamín..., por no citar más que una parte representativa de su elenco. Soca es, entonces, una figura central para las relaciones literarias transatlánticas, entre América Latina y Europa, entre Montevideo y París en particular. Y como ha señalado Sylvia Molloy, La Licorne francesa marca un progreso importante respecto a las revistas latinoamericanas precedentes publicadas en París, en las que no existía un verdadero diálogo intelectual entre los dos continentes. Aunque Susana Soca pagaba y tenía los medios para cuidar la edición, su revista quedaba lejos de las revistas de «ricos latinoamericanos» ${ }^{11}$ o de revistas subvencionadas por gobiernos cuyo objetivo era más bien propagandístico de una literatura nacional y sin un verdadero interés por lo que se hacía en Europa. Revistas que por otro lado se conocían más en América Latina que en Francia.

De hecho, La Licorne nació como una necesidad surgida de unas extremas circunstancias políticas y culturales, en un momento de vacío editorial que más allá del sentido de una urgencia, posibilitó su existencia y le dio su sentido $^{12}$. Ahora bien, como señalé, Susana Soca funda curiosamente su revista

voluntad de ofrecerse -en sus energías, en su tiempo- para una obra a la que le faltó para cumplirse otra generosidad: la de posibles lectores y colaboradores. Susana fue enormemente generosa. Los uruguayos no. Primó el recelo, la costumbre del retaceo, la envidia, el hábito de igualar para abajo. «Por qué traducir escritores europeos si acá está lleno de buenos o mejores.» «Deprimente lugar común.» (entrevista por correo electrónico, diciembre 2004). En este sentido entendemos el comentario de Juan Carlos Onetti (1975), cuando explica su reacción al proponerle Susana Soca publicar un cuento en su revista: «Por entonces yo también estaba influido por el ambiente 'antilicorne'. De modo que pedí un precio increíble para aquellos tiempos y me tomé la mezquina venganza de colocarle un título casi tan largo como una página. Susana pagó agregando su lamento por no ofrecerme más, ya que la revista mostraba un déficit implacable y previsto. El cuento fue publicado sin mutilar el título.» En una carta a Alexis Léger, del 12 de diciembre de 1947, Roger Caillois afirma que La Licorne pagaba 2000 francos por página ( $c f$. I.1.2., p. 45). Incluso si las citas conciernen al periodo uruguayo, la posición de la revista debía ser más ventajosa en Francia al fin de la guerra al contar con el dinero resguardado en Uruguay.

11 Dos ejemplos podrían ser La Revue de l'Amérique latine o la Revue Argentine.

12 La revista no hubiera podido existir en otro lugar ni en otro momento histórico: «El pensamiento de la revista, la intención de hacerla vivir, me apareció bruscamente en forma definida durante los últimos días de la ocupación alemana en París», escribe Susana Soca en el editorial del primer número. Así, desde su intencionalidad primera La Licorne fue un proyecto francés y por consiguiente tenía que ser publicada en Francia. 
el mismo año (1947) en que regresa a Montevideo; se convierte, por lo tanto, paradójicamente, en fundadora y directora de una revista francesa desde la ausencia. Esta particularidad le confiere un lugar bien distinto al del más corriente director de revista omnipresente, ubicuo e impositivo, cuya presencia es imprescindible, como podría ser el caso de Victoria Ocampo en Sur, otra rica rioplatense vinculada a las letras universales y contemporánea a la uruguaya ${ }^{13}$.

Además de sus actividades de mecenas y dinamizadora cultural, también en Francia Susana Soca empezó a escribir poemas y ensayos literarios que, sin embargo, parecen haber quedado en un segundo plano, relegados a su faceta más visible de editora. En efecto, frente a la responsabilidad pública de dirigir una revista, la escritura permaneció en un espacio más íntimo y privado, ya que en vida solo publicó algunos textos en revistas, especialmente en la suya. Su obra solo sería recogida de manera póstuma, en tres libros publicados gracias al impulso de amigos y colaboradores de la revista: En un país de la memoria (1959), Noche cerrada (1962) y Prosas de Susana Soca (1966), los tres bajo el sello La Licorne, convertida en editorial para la ocasión. Se trata de una obra que surgió en realidad bajo las circunstancias precisas de su muerte y que permanecen inaccesibles, puesto que solo de modo muy parcial y circunstancial se la ha vuelto a editar ${ }^{14}$.

Por consiguiente, si como editora actuaba en la distancia y había que reconocer su ausencia como un rasgo original, Susana Soca nace como autora tras su muerte, una evidencia para cualquier autor cuya obra se publica de manera póstuma, pero que en su caso cobra una doble dimensión porque junto a la publicación inmediata de su obra, su muerte provocó también la proyección de su imagen de poeta. En efecto, en 1961, se publica el último número de la revista La Licorne $\left(\mathrm{n}^{\circ} 16,1961\right)$ a modo de homenaje, en el que diferentes autores de distintas nacionalidades, colaboradores habituales de la revista, escriben sobre ella retratándola desde el recuerdo, fijándola en el pasado. Y tras esta serie de textos, se publica una selección de poemas de Susana Soca, haciendo coincidir de este modo el homenaje con la primera presentación pública de sus versos y de su imagen en tanto poeta. Así se inicia un imaginario que acompañará el nombre de Susana Soca, fatalmente identificado con las trágicas circunstancias de su propia muerte, una muerte, por lo demás, muy literaria: en las llamas de un trágico accidente de avión entre París y Montevideo (a ella hacen referencia Jorge Luis Borges y Emilio Oribe en sus poemas, por ejemplo ${ }^{15}$ ). De modo que

13 Pero también de otros directores de revistas de la época, como Jean Paul Sartre en les Temps Modernes o Emmanuel Mounier en Esprit.

14 El Ministerio de Cultura publicó en Uruguay unos pocos ejemplares de uno de esos tres libros en ocasión del centenario de su nacimiento.

15 El poema de Borges (1961: 17) termina: «Dioses que moran más allá del ruego / la abandonaron a ese tigre, el Fuego»; la última estrofa del poema de Emilio Oribe (1961: 26), por su parte, dice: «Si lo absoluto hallaste, / fue en el seno de aquel relámpago en que morías, / y lo permanente / en el lenguaje de tus reinos que ya no existen, / o en la hermosura de las estrellas fugaces / que te miraron / extinguirte, / en la misma 
no solo el carácter póstumo de su obra imposibilitó la participación de Soca en tanto escritora en la sociedad, sino que en tanto autora quedó identificada con el misterio y lo inefable, en un espacio inalcanzable, y por lo tanto doblemente relegada del campo social real de todo escritor; el carácter poético de Soca parece surgir más del efecto de su muerte que de su realidad de escritora.

Como en el caso de otras mujeres -Delmira Agustini es un caso paradigmático de ello en el Uruguay-, la imagen de Susana Soca aparece como «enigma», teñida de misterio, vinculada a su muerte y por eso mismo situándola en un espacio inasible, espiritual, desposeída de las marcas materiales del cuerpo y desconnotada de sexualidad; de modo que no escapa a las interpretaciones biografistas, en general guiadas por las categorías de género, que se han hecho sobre muchas otras mujeres escritoras, cuyas muertes trágicas o escandalosas -si no también sus vidas- determinan la recepción de su obra, la excluyen del canon literario o la relegan a un lugar meramente anecdótico. En el caso de Soca, a la trágica muerte, se suman los conflictos provocados por la desaparición o dispersión de gran parte de su valiosa biblioteca de primeras ediciones (nos consta que contenía ediciones originales de Goehte, por ejemplo), así como de algunos cuadros de su importante pinacoteca (contaba con obras de Picasso, Cézanne, Monet, Sorolla, Modigliani, o De Chirico, entre otros ${ }^{16}$ ). Del mismo modo, llama la atención la negligencia con que se han tratado sus manuscritos y documentos que podrían haber configurado un valioso archivo, hoy inexistente.

Resumiendo y para ir concluyendo, si en tanto mecenas hay una entrega de la autora por fomentar y proteger la obra de sus pares, y en tanto editora está simbólicamente presente a través de la inscripción gráfica de su nombre en la cobertura de la revista, la escritora se hace visible mediante la pluma subjetiva de otros autores y se convierte, en cierto modo, en materia relatable. Escamoteada de su identidad de escritora, queda ligada a la literatura desde el espacio paratextual ${ }^{17}$, engullida por las obras de otros autores, convertida en poema (Borges) o en dedicatoria para una novela (Onetti le dedica el Juntacadáveres: «Para Susana Soca. Por ser la más desnuda forma de piedad que he conocido; por su talento»); también abundan los retratos de artistas que la esculpen, la pintan, la fotografían (Gisèle Freund, Valentine Hugo, Hector Cárdenas, Eduardo Yepes).

En las diferentes actividades a las que se dedicó o los varios roles que ocupó Susana Soca está presente en la tradición paradójicamente desde su ausencia, como una huella que vuelve visible lo que no está, o cual el negativo de una fotografía sin revelar. Desposeída de un lugar de autora en el campo literario, se convierte en patrimonio cultural y social. Susana Soca es el nombre de una calle

llama que las devora a ellas».

16 Jorge Castillo participó en la venta de la colección de Susana Soca, ver la entrevista que le hizo Loustaunau (1999). Allí habla de la colección de ediciones princeps y de cuadros que poseía Soca.

17 Tomo el término del teórico francés Gérard Genette (1987). 
de Montevideo, hace referencia a un premio literario nacional, designa también a una escuela... Es así que como puro nombre, Susana Soca está presente en distintos ámbitos culturales o sociales... de modo que es restituida a la memoria colectiva del Uruguay. Convertida en leyenda, sobre Susana Soca se escucha, se fantasea, se habla, y no parece necesario leer sus textos o consultar su revista para referirse a ella.

En definitiva, desde los márgenes y los intersticios de la tradición, Susana Soca revela los desplazamientos y trasposiciones que tienen lugar en el sistema de producción literaria y cultural, donde los caprichos de la memoria y el olvido, el desorden y las superposiciones, impiden cualquier fijación en una categoría estable, pero dan lugar y hacen siempre posible la creación. Es entre el saqueo y la restitución donde Susana Soca, al fin, se afirma y se carga de sentido precisamente por mostrar las brechas de esa misma tradición.

\section{Bibliografía}

Álvarez Márquez J., 2001, Susana Soca, esa desconocida, Montevideo, Linardi y Risso.

Amengual Cl., 2012, Rara avis. Vida y obra de Susana Soca, Montevideo, Prisa Ediciones.

Borges J.L., 1961, «Susana Soca», Entregas de la Licorne 16, p. 17.

Drieu La Rochelle P., 1992, Journal 1939-1945, Paris, Gallimard.

Estapé J.M.a, 1926, La obra oratoria y literaria del profesor Francisco Soca, Montevideo, Agencia General de Librería y Publicaciones.

Genette G., 1987, Seuils, Paris, Seuil.

Litvan V., 2008, Susana Soca et le champ culturel uruguayen: projet, pratique et image d'écrivain, Tesis de doctorado, Universidad Paris 8.

Loustaunau F., 1999, «El sur del arte uruguayo» [entrevista a Jorge Castillo], El observador, Uruguay, viernes 8 de enero de 1999, p. 3.

Onetti J.C., 1975, «Recuerdo para Susana Soca », Mundo Hispánico (Madrid) 333, p. 64-65.

Oribe E., 1961, «A Susana Soca», Entregas de la Licorne (Montevideo) 16, p. 45-46.

Soca S., 1966, Prosas de Susana Soca, Montevideo, La Licorne.

Torre G. de (1959), «Susana Soca, La Licorne y Pasternak», Sur (Buenos Aires) 257, p. 56-59.

Vannier G., 1988, Histoire de la littérature française: $x x^{e}$ siècle, tome 2: depuis 1945, Paris, Bordas.

Zum Felde, Alberto, 1930, Proceso intelectual del Uruguay y crítica de su literatura, vol. I, Montevideo, Ed. subvencionada por la Comisión Nacional del Centenario. 